\title{
Mathematical Reviews Celebrates 75 Years
}

At the January 2015 Joint Mathematics Meetings in San Antonio, Mathematical Reviews will celebrate its 75 th birthday. There will be a number of celebratory activities, including cake served in the AMS booth at the Exhibits on Sunday, January 11 at 2:30. A number of past executive editors of Mathematical Reviews are expected to attend. Be sure to mark your calendar, if you plan to be in San Antonio.

Volume 1, Number 1 of the paper publication Mathematical Reviews was published in January of 1940. While the monthly paper publication ended its run with the December 2012 issue, Mathematical Reviews is going strong, delivering its content on a daily basis in the electronic service MathSciNet.

The mission of Mathematical Reviews (MR in the following) has remained essentially unchanged over the years: To provide a comprehensive view of the published mathematical research literature under the guidance and supervision of professional mathematicians, both internally as editors, and externally as reviewers. The dedicated reviewers remain a key component of the work of MR.

MR began its work in Providence, Rhode Island in late 1939, and the first issue of Mathematical Reviews appeared surprisingly quickly thereafter, in January of 1940. The founding editor was Otto Neugebauer, well known as an historian of mathematics, and at that time a professor at Brown University in Providence. The founding of Mathematical Reviews was a direct result of the social unrest in Germany in the 1930s associated with Nazism. Neugebauer had also founded the review journal on which Mathematical Reviews was modeled: Zentralblatt für Mathematik. MR has been located

DOI: http://dx.doi.org/10.1090/noti1200 in Ann Arbor, Michigan, since 1965, i.e., for fifty of its seventy-five years. The physical home for MR since 1984, the historic brick building built in 1902 had been a former brewery, back in the days when every US city had multiple breweries. Some interesting reading about the early days of Mathematical Reviews can be found at WwW.ams.org/ pubications/mristoryarticles.

At the start, MR produced a monthly publication in paper. Mathematicians of a certain age associate the orange cover ("Princeton orange") of that publication with MR. As graduate students they were likely taught early on that mathematical investigations should begin with those orange volumes. Lore has it that airport pickups of visiting mathematicians sometimes involved the local host holding an issue of Mathematical Reviews for identification purposes.

The key to the success of MR over the years has been the people involved in producing it, beginning with the reviewers. The reviewers for Volume 1, Number 1 of Mathematical Reviews are a who's who of prominent mathematicians at the time, including Lars Ahlfors, Richard Courant, Paul Erdós, Einar Hille, Alston S. Householder, D. H. Lehmer, Saunders Mac Lane, and John von Neumann, to name just a few. A complete list of the reviewers in that first issue can be found at www. ams .orq/publications/math-reviews/ Revi ewersVolume1. htm7. From a fairly small staff and about 300 reviewers in 1940, MR has grown to a staff of over seventy-five in Ann Arbor and over 17,500 reviewers worldwide. In 2013 reviews were received from 125 countries and at the time of this writing in 2014, reviews have been received from 127 countries. Virtually all of the data and review information found in MathSciNet has been looked at by at least two sets of human eyes, and 
more often by four or more. The consistency and accuracy of the data are guaranteed by the diligence of hardworking people. Although computer software helps those people perform efficiently, literally thousands of human judgments are made every day: judgments ranging from decisions about the inclusion and classification of material in the database, to the unique identification of authors in the MR Author Database, to the nuances of wording in reviews.

The year 1996 marked the launch of MathSciNet, the first web-based electronic presentation of information in the MR Database, although a number of smaller-scale electronic formats had existed before that. In a major digitization effort, the material produced prior to the mid 1980s was incorporated into MathSciNet, so that by 2000 virtually all of the content of paper Mathematical Reviews was available in MathSciNet. The electronic format made new content feasible: linked reference lists from original journal papers beginning in 2000; expanded coverage of applied statistics and applied computer science (Database Expansion items, as they are called) beginning in 2000; entries from pre-1940 digitization projects (DML items) beginning in 2003; citation counts beginning in 2005; and thesis data (ProQuest items) beginning in 2010.

Among scientists, mathematicians have probably been the most interested, historically, in personal connections - witness the Erdós Number and fascination with mathematical ancestry through the Mathematics Genealogy Project (MGP). These personal connections were important from the start of MR, and the work that went into the production of early author indexes made possible the creation of the MR Author Database, with unique identification of authors of mathematical works. In a real sense, the disambiguation of authors that is now a hot topic in academic publication circles has been integral to MR since 1940. This disambiguation work can be seen in the accuracy of author profiles in MathSciNet. These reliable author identifications allow not only the calculation of Erdôs Numbers, but the determination of the co-author distance between any two authors in the database. They make possible the pairing of MR and MGP data, which has been an important addition to MathSciNet.

At the Joint Mathematics Meetings in Baltimore in January, 2014, the personalization of MathSciNet author profile pages was released. Authors can now log into their MathSciNet profile pages and add one or more of the following: a photo, an email address, a personal URL. A little reward (a bit of meeting "swag") awaits anyone who stops by the MR Booth in San Antonio to show off their author profile personalization.

All friends of MR are cordially invited to the annual Mathematical Reviews Reception, Monday,
January 12, 2015, from 6:00 to 7:00 pm, in Bowie B, 2nd Level, Grand Hyatt, San Antonio. There will be a number of MR staff members present, including the recently appointed Executive Editor, Edward Dunne, who is the twenty-first Executive Editor. As was already mentioned, a number of past Executive Editors are expected to attend the JMM and will be at the reception. The complete list of Executive Editors can be found at www.ams.org/ pubications/math-reviews/executiveeditors.

Anyone who visits Ann Arbor is encouraged to stop in for a visit. The MR offices are located in an historic neighborhood called the Old West Side, with many beautifully restored houses and buildings. With a day or two of advance notice, a tour can be arranged. Just send a message to mathrev@ams.org. Some photos and some building history can be found at www. ams.org/ publications/mrbuildingphotos, more on the history of MR at Www. ams.org/publications/ math-reviews/mrpastandpresent, and a video about MR and its history at www. youtube.com/ watch?v=cUEOghB3MvM.

- Norman Richert, Managing Editor Mathematical Reviews/MathSciNet

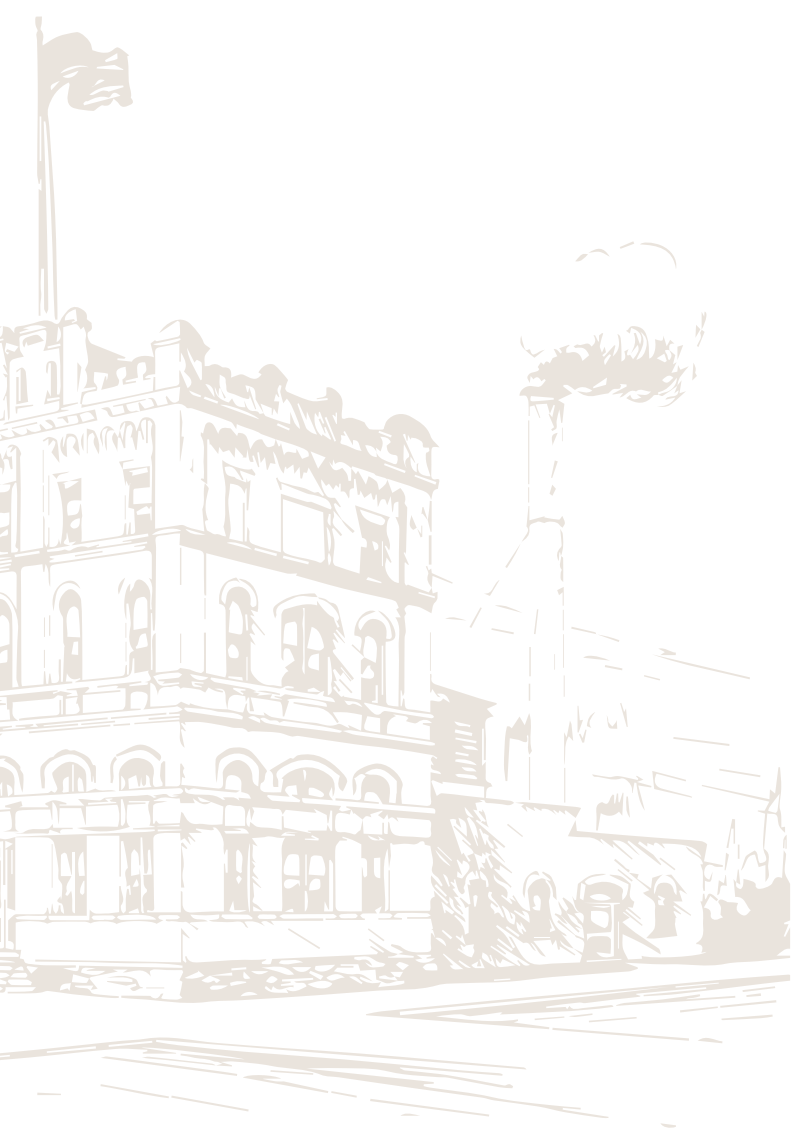

